\title{
A Previously Unknown Antibiotic Complex from the Fungus Cyathus helenae
}

A previously undescribed Bird's Nest fungus was recently discovered and named Cyathus helenae Brodie ${ }^{2}$. OLCKOWECKI ${ }^{3}$ was the first to observe that cultures of the new fungus checked the growth of bacteria when some cultures accidentally became contaminated. A few preliminary tests of the antimicrobial action of the filtrate were made. Further investigation of the antibiotic activity of culture filtrate of $C$. helenae has been carried out and is the subject of this preliminary report.

A completely defined liquid medium with the following composition was developed for growing the fungus under static conditions : dextrose, $30 \mathrm{~g}$; asparagine, $1.5 \mathrm{~g}$; potassium phosphate (dibasic), $1 \mathrm{~g}$; calcium nitrate, $0.5 \mathrm{~g}$; magnesium sulphate, $0.5 \mathrm{~g}$; zinc sulphate, $0.25 \mathrm{mg}$; thiamine, $0.15 \mathrm{mg}$; distilled water to make 11 . The brownish antibiotic complex tentatively named cyathin, was isolated from the culture filtrate by extraction with ethyl acetate. Maximum yield was usually obtained in 22 to 25 days at $22-25^{\circ} \mathrm{C}$. Physical factors of the environment appeared not to alter the yield appreciably. When different combinations of carbon and nitrogen sources were used, it was found that the proportions of the various components of the cyathin were altered. Cyathin is heat-stable, relatively $\mathrm{pH}$ stable, and is not highly soluble in water. It has a low diffusion rate on agar.

The cyathin complex has a broad spectrum of antimicrobial activity. It was found to inhibit the growth of actinomycetales, gram-positive and gram-negative bacteria, and fungi including 'dermatophytes'.

Not all cultures of $C$. helenae were found to produce the cyathin complex. Moreover, many single-spore cultures were found to undergo marked sectoring ${ }^{4}$ and isolates from various sectors varied in the yield of cyathin. An unexpected observation was that apparently only single-spore monokaryon mycelia of $C$. helenae produced cyathin; to date no dikaryon mycelium has been observed to be biologically active.

Thin-layer chromatography on silica gel revealed that the complex consisted of several components. By means of preparative thin-layer chromatography and column chromatography 7 active substances have been obtained and their apparent molecular formulae have been determined ${ }^{5}$ as follows: cyathin $\mathrm{A}_{3}\left(\mathrm{C}_{20} \mathrm{H}_{30} \mathrm{O}_{3}\right)$, IR-spectrum: $3580,3400,1710,1650 \mathrm{~cm}^{-1}$; cyathin $\mathrm{A}_{4}\left(\mathrm{C}_{20} \mathrm{H}_{30} \mathrm{O}_{3}\right)$, IRspectrum: $3600,3400,1700(\mathrm{w}), 1650(\mathrm{~m}), 1375 \mathrm{~cm}^{-1}$; allocyathin $\mathrm{A}_{4}\left(\mathrm{C}_{20} \mathrm{H}_{30} \mathrm{O}_{4}\right)$, IR-spectrum: $3600,3490,1710$ (broad) $\mathrm{cm}^{-1}$; cyathin $\mathrm{B}_{3}\left(\mathrm{C}_{20} \mathrm{H}_{28} \mathrm{O}_{3}\right)$, IR-spectrum: 3590, $1680 \mathrm{~cm}^{-1}$; cyathin $\mathrm{B}_{4}\left(\mathrm{C}_{20} \mathrm{H}_{28} \mathrm{O}_{4}\right)$, IR-spectrum: 3570 ,
$1675,1595 \mathrm{~cm}^{-1}$; cyathin $\mathrm{C}_{5}\left(\mathrm{C}_{20} \mathrm{H}_{26} \mathrm{O}_{5}\right)$, IR-spectrum: $3400,3200,1695,1675,1235,1220 \mathrm{~cm}^{-1}$; chromocyathin $\left(\mathrm{C}_{7} \mathrm{H}_{6} \mathrm{O}_{4}\right)$, IR-spectrum: $3380,3160,1630,1610,1590 \mathrm{~cm}^{-1}$. When these compounds were tested for antimicrobial activity, all proved to be active although there were some differences in the action spectrum and biologically effective concentrations.

It has been shown that chromocyathin is identical with $2,4,5$-trihydroxybenzaldehyde ${ }^{6}$. This substance has been synthesized from hydroxyhydroquinone using the ADAMs' modification ${ }^{8}$ of the GATTERMAN synthesis ${ }^{7}$ and the synthetic product found to have antimicrobial activity.

Studies of the structure of the $\mathrm{C}_{20}$ compounds are in progress. These compounds appear to be closely related members of a new group of diterpenoids, and elucidation of their structure is of interest from a chemical as well as a biological point of view ${ }^{8}$.

Résume. Cyathus helenae produit un complexe antibiotique à action générale. Le complexe comprend 7 constituants qui ont été séparés par chromatographie en couche mince et dont les formules moléculaires ont été determinées. Les constituants possèdent 20 atomes de carbone à l'exception d'un seul dont la formule est $\mathrm{C}_{7} \mathrm{H}_{6} \mathrm{O}_{4}$.

\section{B. N. Johri ${ }^{9}$, H. J. Brodie, A. D. Allbutt,} W. A. Ayer and H. TAUBE

Department of Botany and Department of Chemistry, University of Alberta, Edmonton 7 (Alberta, Canada), 12 January 1971.

1 Based on a Ph.D. thesis submitted by B. N. Johri to the University of Alberta, May 1969.

2 H. J. Brodie, Can. J. Bot. 44, 1235 (1966).

A. Otchoweckr, Culture studies and mating reaction in Cyathus helenae Brodie and related species. Master's thesis, University of Alberta, September 1967.

4 A. Olchowecki and H. J. Brodie, Can. J. Bot. 46, 1423 (1968).

5 Molecular formulae were determined by combustion analysis and high resolution mass spectrometry.

- R. Adams and I. Levin, J. Am. chem. Soc. 45, 2373 (1923).

7 L. Gatterman and M. KöBnER, Chem. Ber. 32, 278 (1899).

8 This study was supported by funds provided by the National Research Council of Canada.

- Present address: Department of Botany, University of British Columbia, Vancouver 8 (British Columbia, Canada).

\section{Effets immédiats de quelques antibiotiques sur l'électro-osmose chez Nitella flexilis $\mathbf{L}$.}

Chez Nitella, l'électro-osmose est conditionnée principalement par la structure de la membrane cellulaire. Celle-ci serait percée de pores chargés négativement, remplis d'eau ${ }^{1-3}$. Les variations du flux d'eau électroosmotique en présence de substances ajoutées au milieu peuvent éventuellement trahir les interactions de celles-ci avec les composantes de la membrane cellulaire ou encore avec des processus métaboliques intervenant dans ce phénomène. Dans ce but, nous avons testé l'effet de trois antibiotiques, choisis pour leur mode d'action différent sur la cellule ${ }^{4}$. Il s'agit du chloramphénicol, un inhibiteur de la synthèse protéinique, de l'oligomycine, un découplant des phosphorylations oxydatives et de la polymyxine, un composé cationique actif sur la surface membranaire.

Les expériences ont porté sur Nitella flexilis L., recueillie dans une ancienne carrière à Wood Point N.B. (Canada). Au moment de l'essai, une cellule internodale est isolée du télome et placée dans un électro-osmomètre de même type que celui décrit par Fensom et Dainty ${ }^{1}$. L'ensemble est plongé dans un bain thermostabilisé à $23^{\circ} \mathrm{C}$. Les deux compartiments de l'électro-osmometre, l'extérieur débouchant directement à l'air et l'intérieur, via un capillaire, sont remplis d'une solution minérale (APW) dont la composition est la suivante: $0,1 \mathrm{mM} \mathrm{KCl}$, $1,0 \mathrm{~m} M \mathrm{NaCl}$ et $0,1 \mathrm{mM} \mathrm{CaCl}{ }_{2}$. Le mouvement d'eau en 